\title{
Mandibular advancement devices in the treatment of obstructive sleep apnea and snoring in community dental care: A pilot study on self-reported sleep quality
}

\author{
${\text { Tiina-Riitta Vuorjoki-Ranta }{ }^{1^{*}}, \text { Frank Lobbezoo }^{2} \text {, Henri Tuomilehto }}^{3,4}$, Mauno Könönen ${ }^{5}$, \\ Antti Pihakari ${ }^{1}$, Jari Ahlberg ${ }^{1,5}$

\footnotetext{
${ }^{1}$ Helsinki Health Centre, Unit for Specialized Oral Care in the Metropolitan Area and Kirkkonummi, Helsinki, Finland;

*Corresponding Author: t.vuorjoki@kolumbus.fi

${ }^{2}$ Department of Oral Kinesiology, Academic Centre for Dentistry Amsterdam (ACTA), Research Institute MOVE, University of Amsterdam and VU University, Amsterdam, Netherlands

${ }^{3}$ Department of Otorhinolaryngology, Kuopio University Hospital, Kuopio, Finland

${ }^{4}$ Oivauni Sleep Clinic, Kuopio, Finland
} \\ ${ }^{5}$ Department of Stomatognathic Physiology and Prosthetic Dentistry, Institute of Dentistry, University of Helsinki, Helsinki, Finland
}

Received 30 May 2013; revised 30 June 2013; accepted 15 July 2013

Copyright (C) 2013 Tiina-Riitta Vuorjoki-Ranta et al. This is an open access article distributed under the Creative Commons Attribution License, which permits unrestricted use, distribution, and reproduction in any medium, provided the original work is properly cited.

\begin{abstract}
Background: The high efficacy of continuous positive airway pressure (CPAP) in treating obstructive sleep apnea (OSA) patients is sometimes limited because of intolerance. Mandibular advancement devices (MAD) are proven to be efficient in treating mild to moderate sleep apnea and snoring. We examined patients who had been treated in Community Dental Care with a titrable Herbst-type MAD. The aim of the study was to evaluate the quality of sleep based on self report. Methods: A questionnaire was mailed to patients $(n=184)$ who had received treatment for OSA or snoring between 2007 and 2010 in the Helsinki Health Centre Oral Care Unit. The patients were referred to the clinic for primary treatment, or because another treatment modality had failed. Results: Of the respondents ( $\mathrm{n}=$ $142,78.4 \%$ ), OSA had been diagnosed in $74 \%$, while the MAD was applied for snoring to the remaining. Among all respondents, $78.4 \%$ had worn the MAD at least three nights per week. With the MAD in situ, sleep was felt significantly less disrupted $(p<0.001)$ and more restorative ( $p<0.001$ ), and snoring was markedly reduced $(p<0.001)$. Daytime tiredness was also markedly less frequent $(p<0.001)$. Conclusions: Treatment with a MAD improved perceived sleep
\end{abstract}

quality and awoke alertness in mild and moderate OSA patients and in snorers. This study supports such treatment to be initiated and monitored in community dental care.

Keywords: Obstructive Sleep Apnea; Oral Appliance; Compliance; Daytime Sleepiness; Snoring; Community Dental Care

\section{INTRODUCTION}

Obstructive sleep apnea syndrome (OSA) is a clinically diagnosed sleep disorder with a reported prevalence about $2 \%$ in women and $4 \%$ in men $[1,2]$, but its actual prevalence may be higher. In the etiology of the syndrome, obesity is the most prevalent risk factor for OSA. Reportedly, $60 \%-70 \%$ of OSA patients are overweight. It is worth of notion, however, that in about $30 \%$ are of normal weight. Recent research has indicated that variation in craniofacial anatomy and occlusion should be borne in mind as possible additional etiological factors for OSA [3].

The use of oral appliances (OAs) in the treatment of obstructive sleep apnea (OSA) and snoring has increasingly become an alternative for continuous positive airway pressure (CPAP); the latter still being the gold standard because of its proven effectiveness [4]. Recent research, however, has narrowed the gap between OAs and CPAP $[5,6]$. Yet, oral appliances seem less effective in 
reducing the apnea-hypopnea index (AHI) when both treatment modalities are being regularly used. However, due to their better compliance and effect on respiratory events in position dependent OSA, OAs may even be a better treatment option in some patients with mild or moderate OSA [7].

Measuring AHI, Aarab et al. [5] found no difference in efficacy between OA and CPAP when the mandibular advancement device (MAD) was titrated individually, which is also the standard procedure when initiating treatment with CPAP. Both treatment modalities showed significantly better effects than a placebo device. Their RCT is in fact the first to report results with such titration efforts to accomplish an unbiased study. Most recent studies have focused on the clinical outcomes over subjective experiences, but Aarab et al. [5] also showed a good compliance and only mild side effects in the MAD group.

The treatment of OSA is demanding for both patients and health care personnel, because there are no simple treatment modalities. There is a clear need to clarify the clinical guidelines and, thus, to identify novel well tolerated and cost-effective treatment options for OSA patients as well as to improve the existing treatment modalities.

Thus, due to lack of knowledge of using MADs in practical care, the present study aimed to evaluate in the Community Dental Care setting self-reported MAD treatment-related compliance and sleep quality in patients with mild-to-moderate OSA.

\section{MATERIAL AND METHODS}

In 2010, a questionnaire was mailed to patients who had received treatment for OSA or snoring between 2007 and 2010 in the Helsinki Health Centre Oral Care Unit (n $=184$ ). All patients were treated with a titrable Herbsttype mandible advancement device (MAD) (Figure 1), which was fabricated free of charge. All patients were treated by one of the authors (AP), and all devices were fabricated by the same dental technician. The patients were referred to the clinic for primary treatment, or be-

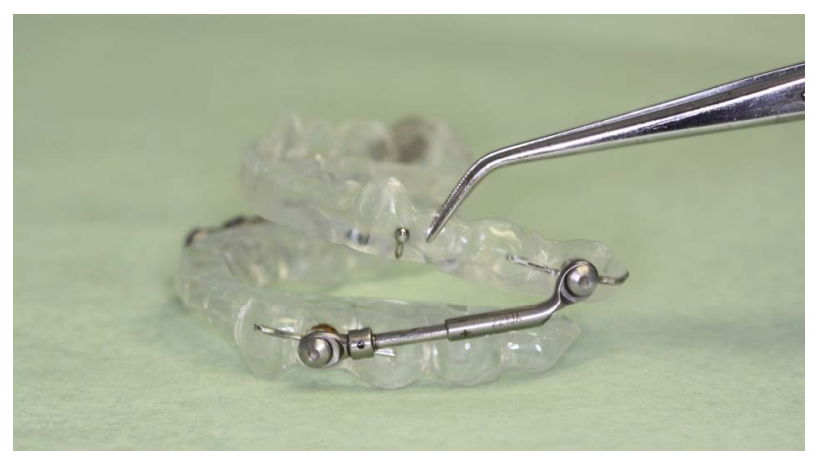

Figure 1. Herbst-type titrable mandibular advancement (MAD) device used in the present study. cause previously made ENT surgery had not helped their symptoms. Some patients' CPAP treatment had failed due to, e.g., poor compliance because of side effects or other things.

From the questionnaire, data were used and classified for the present study as follows:

1) descriptive data: gender, age, weight, length, waist and neck circumference (a measuring tape was attached to the questionnaire and respondents were instructed to measure neck circumference just below the laryngeal prominence), tobacco use (current smoker vs. non-smoker), alcohol consumption (equivalent doses of wine, beer, or spirits), aspects of general health, and orofacial pain experience during the past month [8-11]. The body mass index (BMI) was calculated from the weight and length data.

2) compliance in using the oral appliance: self-assessed frequency of wearing the MAD (never or less than once a month, less than weekly, 1 - 2 nights per week, 3 - 5 nights per week, every night or almost every night).

3) insomnia symptoms [12,13]: difficulties initiating sleep (DIS), disrupted sleep (DS), and early morning awakenings (EMA) (at least 3 nights per week).

4) perceived consequences of sleep: non-restorative sleep (NRS) (sustained $>1$ month), and tiredness (at least 3 days per week).

5) snoring: as perceived or reported by a bed partner (at least 3 nights per week).

\section{STATISTICAL METHODS}

The Chi square test was used to study the associations between categorical variables. Student's $t$ test was used to compare group means. McNemar's change-test was used to compare the insomnia symptoms and perceived consequences of sleep both without and with the MAD in situ. Since there was no gender difference in the occurrence of these symptoms, the data of both genders were pooled. The level of statistical significance was set at $p<$ 0.05 .

\section{RESULTS}

The response rate was $78.4 \%(n=142 ; 66 \%$ men $)$. Mean age for men was 51 years (SD 11) and for women 58 years (SD 11). BMI was not associated with gender. In both genders, $24 \%$ were current smokers. Without gender difference either, $41 \%$ of the subjects considered their health as "good" or "very good", and only two per cent reported their health as very poor. Neither was diagnosed illnesses related with gender; most prevalent of them were hypertension blood pressure $(35 \%)$, allergic rhinitis $(25 \%)$, and cardiac arrhythmia (18\%). Altogether, $22 \%$ reported orofacial pain during the past month. Table 
1 shows descriptive data.

OSA had been diagnosed in $74 \%$, and the MAD was applied for snoring to the rest. Previous CPAP treatment attempt was reported by $29 \%$, and $40 \%$ had received ENT surgery for their problems of sleep-disordered breathing.

Among all subjects, $78.4 \%$ had worn the MAD at least three nights per week, whereas $10 \%$ reported that they had done so less than weekly. Orofacial pain experience was not associated with the use of MAD.

With the MAD in situ, sleep was experienced to be significantly less disrupted $(p<0.001)$. Similarly, sleep was significantly more restorative $(p<0.001)$, and snoring was markedly reduced $(p<0.001)$. Table 2 shows the occurrence of perceived insomnia symptoms and their consequences without and with the MAD in situ.

\section{DISCUSSION}

The main findings of the present pilot study were that treatment with standard method MAD [5,6,14-16] significantly improved the OSA patients' sleep quality, leading to subjectively better awake alertness. In fact, with the MAD in situ, the patients reported disrupted sleep close to the figures earlier reported in Finland among healthy populations [13]. Furthermore, snoring was also markedly reduced.

A good compliance of MAD has been shown in several studies $[6,7,17-19]$. This finding is supported by our study. The results revealed an interesting triangle, viz., the good subjective effect of MAD treatment yielded a good compliance, bearing in mind the fact that the treatment was provided free of charge, which raised the question why anyone would bother to wear a costless device without experiencing a genuine subjective benefit.
Using polysomnography, Gauthier et al. [20] demonstrated in their follow-up study that the positive effect of MAD on disturbed breathing remained after $2.5-4.5$ years of regular use. Also, a good effect on sleep quality (deep stages 3 - 4 increased) was found. According to these authors, MAD was well tolerated: $94 \%$ of subjects continued using the MAD.

Considering the increasing incidence of sleep-disordered breathing among populations [21], effective treatment modalities are needed to target the treatment of this condition cost-effectively. OSA has a tendency to progress from mild sleep disordered breathing to more severe OSA over a varying period of time, which may be surprisingly short in case of weight gain and lack of effective treatment [22]. Therefore, initiating treatment at an early stage of OSA could be beneficial to the prevention of the development of more severe problems. MAD has recently been shown equally effective compared with CPAP when both devices are titrated individually [5,7]. Thus, in normal-weight patients with mild and moderate OSA, and especially in the case of disturbing snoring, MAD could perhaps be the first-choice treatment strategy.

Prerequisitely, in order to successfully apply MAD, treatment necessitates a suitable dentition and craniofacial structure, i.e., healthy periodontium, sufficient number of teeth in both arches and no severe class III occlusion. Thus, dentists play a key role in the protocol. Dentists are trained to fabricate these devices and are able to control possible dental complications. In addition, during a standard dental examination, some oral and craniofacial landmarks of possible predisposing factors of OSA could easily be detected. Since the dentists are an important group to meet with undiagnosed sleep-disordered

Table 1. Demographic data on the study population by gender and health experience. Student's t-test.

\begin{tabular}{|c|c|c|c|c|c|c|}
\hline \multicolumn{4}{|c|}{ Gender } & \multicolumn{2}{|c|}{ Health experience } & \\
\hline & Male & Female & & good/very good & else $^{*}$ & \\
\hline \multirow[t]{2}{*}{$\mathrm{n}=$} & 93 & 49 & & 58 & 84 & \\
\hline & mean (SD) & & $\mathrm{p}=$ & mean (SD) & & $\mathrm{p}=$ \\
\hline Age (yr) & $50.7(11.4)$ & $57.8(11.4)$ & 0.001 & $53.1(12.6)$ & $53.2(10.9)$ & 0.991 \\
\hline Waist circumference $(\mathrm{cm})$ & $101.5(10.2)$ & $92.3(12.7)$ & $<0.001$ & $94.2(9.7)$ & $101.3(12.6)$ & $<0.001$ \\
\hline Neck circumference $(\mathrm{cm})$ & $41.9(3.1)$ & $37.1(3.5)$ & $<0.001$ & $39.2(3.8)$ & $41.0(3.9)$ & 0.018 \\
\hline BMI & $27.8(4.4)$ & $27.2(6.3)$ & 0.569 & $25.6(3.5)$ & $28.9(5.6)$ & $<0.001$ \\
\hline Alcohol consumption & $7.8(7.3)$ & $3.3(4.7)$ & $<0.001$ & $6.7(6.9)$ & $5.9(6.7)$ & 0.513 \\
\hline Current smoking $^{1}$ & $24.7(\%)$ & $22.9(\%)$ & 0,811 & $17.5(\%)$ & $28.6(\%)$ & 0.095 \\
\hline
\end{tabular}

${ }^{*}$ rather good/ rather poor/ poor/ very poor. 
Table 2. Overall occurrence of perceived insomnia symptoms and their awake consequences without and with MAD in situ. McNemar change test.

\begin{tabular}{|c|c|c|c|c|}
\hline$n=142$ & $\%$ & Without MAD & With MAD & $\mathrm{p}=$ \\
\hline DIS & & 10.6 & 11.3 & 0.500 \\
\hline DS & & 66.9 & 45.8 & $<0.001$ \\
\hline EMA & & 16.9 & 14.1 & 0.424 \\
\hline NRS & & 52.8 & 9.2 & $<0.001$ \\
\hline Tiredness & & 69.7 & 14.1 & $<0.001$ \\
\hline Snoring & & 87.3 & 21.1 & $<0.001$ \\
\hline
\end{tabular}

DIS: difficulties initiating sleep, DS: disrupted sleep, EMA: early morning awakening. NRS: non-restorative sleep.

breathing patients, it should be included in the normal dental examination protocol to ask the patients about their sleeping habits and snoring, especially in the cases with overweight and/or morning headaches. Adding an anamnestic history to the clinical findings could point to a possible sleep-related breathing problem and, when necessary, the patient could be referred to a medical specialist for further examination and diagnostics. It is unclear, however, whether dentists in general are aware of, or prepared for this task.

It is not only important to know how to treat a sleepdisordered breathing patient with a MAD, but it is also essential to establish how to diagnose the condition. In a case of simple snoring in a normal weight person without any other symptoms or other medical conditions diagnosed by a physician, MAD may be considered to be initiated without any further investigations. However, in a case of a suspected OSA, at least ambulatory cardiorespiratory recording, viz. polysomnography with a portable device, is needed to confirm the medical diagnosis $[12,14,15]$. An appropriate verification of the condition may often also increase the compliance for the treatment when the patient is aware of the underlying reason for his/her symptoms.

On the societal level, early intervention of OSA seems an increasing challenge. To further develop the clinical guidelines and thus, to offer better treatment for OSA patients, there is a need to improve the existing treatment modalities and to search for new ones. Earlier beliefs that lifestyle changes would not be efficient in the long run when treating overweight OSA patients may not in the light of recent research be justified $[23,24]$. This should be noted. However, as one-third of OSA patients are not overweight, MADs should be borne in mind as a potential treatment option. As indicated in the present study, such treatment could easily be performed and monitored in the community dental care setting upon medical indication. Further investigation on MADs is needed to monitor the longer term effects of the treatment on OSA, and to find possible oral complications, maintenance need and long service of the device.

\section{REFERENCES}

[1] Young, T., Palta, M., Dempsey, J., Skatrud, J., Weber, S. and Badr. S. (1993) The occurrence of sleep-disordered breathing among middle-aged adults. The New England Journal of Medicine, 328, 230-235. doi:10.1056/NEJM199304293281704

[2] Patil, P.S., Schneider, H., Schwartz, A.R. and Smith, P.L. (2007) Adult obstructive sleep apnea pathophysiology and diagnosis. Chest, 132, 325-337. doi:10.1378/chest.07-0040

[3] Pahkala, R., Puustinen, R., Tuomilehto, H., Ahlberg, J. and Seppä, J. (2011) Risk factors for sleep-disordered breathing: The role of craniofacial structure. Acta Odontologica Scandinavica, 69, 137-143. doi: $10.3109 / 00016357.2010 .545033$

[4] Giles, T.L., Lasserson, T.J., Smith, B., White, J., Wright, J.J. and Cates C.J. (2006) Continuous positive airway pressure for obstructive sleep apnoea in adults. Cochrane Database of Systematic Reviews, CD001106.

[5] Aarab, G., Lobbezoo, F., Hamburger, H.L. and Naeije, M. (2011) Oral appliance therapy versus nasal continuous positive airway pressure in obstructive sleep apnea: A randomized, placebo-controlled trial. Respiration, 81, 411-419. doi:10.1159/000319595

[6] Aarab, G., Lobbezoo, F., Heymans, M.W., Hamburger, H.L. and Naeije, M. (2011) Long-term follow-up of a randomized controlled trial of oral appliance therapy in obstructive sleep apnea. Respiration, 82, 162-168. doi: $10.1159 / 000324580$

[7] Randerath, W.J., Heise, M., Hinz, R. and Ruehle, K.H. (2002) An individually adjustable oral appliance vs continuous positive air pressure in mild-to-moderate obstructive sleep apnea syndrome. Chest, 122, 569-575. doi:10.1378/chest.122.2.569

[8] Partinen, M. and Gislason, T. (1995) Basic nordic sleep questionnaire (BNSQ): A quantitated measure of subjective sleep complaints. Journal of Sleep Research, 4, 150-155. doi:10.1111/j.1365-2869.1995.tb00205.x

[9] Ohrbach, R., List, T., Goulet, J.P. and Svensson, P. (2010) Recommendations from the international consensus workshop: Convergence on an orofacial pain taxonomy. Journal of Oral Rehabilitation, 37, 807-812. doi:10.1111/j.1365-2842.2010.02088.x

[10] Dixon, D., Pollard, B. and Johnston, M. (2007) What does the chronic pain grade questionnaire measure? Pain, 130, 249-253. doi:10.1016/j.pain.2006.12.004

[11] Von Korff, M., Ormel, J., Keefe, F.J. and Dworkin, S.F. (1992) Grading the severity of chronic pain. Pain, 50, 133-149. doi:10.1016/0304-3959(92)90154-4

[12] American Academy of Sleep Medicine (AASM) (1997) The international classification of sleep disorders revised: Diagnostic and coding manual (ICSD). American Acad- 
emy of Sleep Medicine, Rochester.

[13] Ohayon, M.M. and Partinen, M. (2002) Insomnia and global sleep dissatisfaction in Finland. Journal of Sleep Research, 11, 339-346. doi:10.1046/j.1365-2869.2002.00317.x

[14] Flemons, W.W. (2002) Clinical practice. Obstructive sleep apnea. The New England Journal of Medicine, 347, 498-504. doi:10.1056/NEJMcp012849

[15] Epstein, L.J., Kristo, D., Strollo Jr., P.J., Friedman, N., Malhotra, A., Patil, S.P., et al. (2009) Clinical guideline for the evaluation, management and long-term care of obstructive sleep apnea in adults. Journal of Clinical Sleep Medicine, 5, 263-276.

[16] Gauthier, L., Laberge, L., Beaudry, M., Laforte, M., Rompré, P.H. and Lavigne, G.J. (2009) Efficacy of two mandibular advancement appliances in the management of snoring and mild-moderate sleep apnea: A cross-over randomized study. Sleep Medicine, 3, 329-336. doi:10.1016/j.sleep.2008.03.011

[17] Ferguson, K.A., Cartwright, R., Rogers, R. and SchmidtNowara, W. (2006) Oral appliances for snoring and obstructive sleep apnea: A review. Sleep, 29, 244-262.

[18] Lim, J., Lasserson, T.J., Fleetham, J. and Wright, J.J. (2006) Oral appliances for obstructive sleep apnea: Intervention review. Cochrane Database of Systematic Reviews, CD004435.

[19] Barnes, M., McEvoy, R.D., Banks, S., Tarquinio, N., Murray, C.G., Vowles, N., et al. (2004) Efficacy of positive airway pressure and oral appliance in mild to moder- ate obstructive sleep apnea. American Journal of Respiratory and Critical Care Medicine, 170, 656-664. doi:10.1164/rccm.200311-15710C

[20] Gauthier, L., Laberge, L., Beaudry, M., Laforte, M., Rompré, P.H. and Lavigne, G.J. (2011) Mandibular advancement appliances remain effective in lowering respiratory disturbance index for 2.5 - 4.5 years. Sleep Medicine, 12, 844-849. doi:10.1016/j.sleep.2011.05.004

[21] McClean, K.M., Kee, F., Young, I.S. and Elborn, J.S. (2008) Obesity and the lung: 1. Epidemiolgy. Thorax, 63, 649-654. doi:10.1136/thx.2007.086801

[22] Sahlman, J., Pukkila, M., Seppä, J. and Tuomilehto, H. (2007) Evolution of mild obstructive sleep apnea after different treatments. Laryngoscope, 117, 1107-1111. doi:10.1097/MLG.0b013e3180514d08

[23] Tuomilehto, H., Gylling, H., Peltonen, M., Martikainen, T., Sahlman, J., Kokkarinen, J., et al. (Kuopio Sleep Apnea Group) (2010) Sustained improvement in mild obstructive sleep apnea after a diet- and physical activitybased lifestyle intervention: Postinterventional follow-up. The American Journal of Clinical Nutrition, 92, 688-696. doi:10.3945/ajen.2010.29485

[24] Tuomilehto, H., Peltonen, M., Partinen, M., Lavigne, G., Eriksson, J.G., Herder, C., et al. (Finnish Diabetes Prevention Study Group) (2009) Sleep duration, lifestyle intervention, and incidence of type 2 diabetes in impaired glucose tolerance: The finnish diabetes prevention study. Diabetes Care, 32, 1965-1971. doi:10.2337/dc08-1980 